\title{
DESAFIOS DA ENFERMAGEM EM UMA UNIDADE DE TRANSPLANTES ANTE A COVID-19
}

\author{
Nursing challenges in a transplant unit in the face of Covid-19
}

\author{
Retos de la enfermería en una unidad de trasplante frente a Covid-19 \\ Heloisa Sousa Oliveira ${ }^{1 *}$ (D), Alan Rodrigues da Silva² (1), Aglauvanir Soares Barbosa ${ }^{3}$ (D), \\ Isakelly de Oliveira Ramos ${ }^{4}$ (D), Rita Mônica Borges Studart ${ }^{5}$ (1)
}

RESUMO: Objetivo: Conhecer a percepção dos enfermeiros que atuam em uma unidade de transplantes sobre os desafios de sua atuação ante a COVID-19. Método: Estudo descritivo e exploratório, com abordagem qualitativa, desenvolvido em uma unidade de transplantes de um hospital referência do Ceará, entre março e junho de 2020, por meio de entrevista. Os dados foram analisados pelo software Interface de R pour les Analyses Multidimensionnelles de Textes et de Questionnaires (IRAMUTEQ). Posteriormente, foi realizada a análise fatorial por correspondência (AFC) e gerada uma nuvem de palavras para a qual foram consideradas as evocações que apareceram com maior frequência. Resultados: Participaram do estudo 14 enfermeiros, a maioria do sexo feminino. O conteúdo analisado foi categorizado em três classes: organização do fluxo de atendimento de casos suspeitos, equipamento de proteção individual no contexto da pandemia da COVID-19, o emocional ante a pandemia. Conclusão: Constatou-se que os enfermeiros sofrem ante as fragilidades relacionadas ao momento vivenciado, pois estão sendo cotidianamente desafiados a se adaptarem às mudanças em suas rotinas de trabalho. Palavras-chave: Enfermagem. Coronavírus. Transplante.

ABSTRACT: Objective: To understand how nurses who work in a transplant unit perceive the challenges of their role in the face of COVID-19. Method: This is an exploratory, descriptive, qualitative study conducted through interviews in the transplant unit of a reference hospital in Ceará, Northeastern Brazil, between March and June 2020. Data were analyzed in the software Interface de R pour les Analyses Multidimensionnelles de Textes et de Questionnaires (IRAMUTEQ). Subsequently, we performed a correspondence factor analysis (CFA) and generated a word cloud, taking into account the evocations with higher frequency. Results: Fourteen nurses participated in the study, most of them women. The content analyzed was categorized into three classes: organization of the care flow for suspected cases, personal protection equipment in the COVID-19 pandemic context, emotional state in the face of the pandemic. Conclusion: We found that nurses are suffering due to fragilities related to the moment they are experiencing, as they are daily challenged to adapt to changes in their work routines. Keywords: Nursing. Coronavirus. Transplantation.

RESUMEN: Objetivo: Conocer la percepción de los enfermeros que laboran en una unidad de trasplante sobre los desafíos de su desempeño ante el COVID19. Método: Estudio descriptivo y exploratorio, con abordaje cualitativo, desarrollado en una unidad de trasplante de un hospital de referencia en Ceará, entre marzo y junio de 2020, mediante entrevista. Los datos fueron analizados por el software IRAMUTEQ (Interface de R pour les Analyses Multimensionnelles de Textes et de Questionnaires). Posteriormente, se realizó el Análisis Factorial por Correspondencia (AFC) y se generó un Word Cloud para el que se consideraron las evocaciones que aparecían con mayor frecuencia. Resultados: participaron del estudio 14 enfermeras, la mayoría mujeres. El contenido analizado se categorizó en tres clases: Organización del Flujo de Servicios de Casos Sospechosos, Equipo de Protección Personal en el Contexto de la Pandemia de COVID-19, La Emocional Frente a la Pandemia. Conclusión: Se encontró que los enfermeros padecen las debilidades relacionadas con el momento vivido, ya que diariamente se enfrentan al desafío de adaptarse a los cambios en sus rutinas laborales.

Palablas clave: Enfermería. Coronavirus. Trasplante.

Enfermeira. Residente Multiprofissional em Transplante de Órgãos e Tecidos do Hospital Geral de Fortaleza - Fortaleza (CE), Brasil.

Farmacêutico. Mestrando em Transplante pela Universidade Estadual do Ceará. Residente multiprofissional em Transplante de Órgãos e Tecidos no Hospital Geral de Fortaleza - Fortaleza (CE), Brasil. ${ }^{3}$ Enfermeira, Mestre em Enfermagem pela Universidade da Integração Internacional da Lusofonia Afro-Brasileira. Coordenadora de Enfermagem no Centro Cirúrgico Eletivo do Hospital Geral de Fortaleza Fortaleza (CE), Brasil.

“Enfermeira. Mestre em Tecnologia e Inovação em Enfermagem pela Universidade de Fortaleza. Enfermeira assistencial na Unidade de Pós-operatório de Alta Complexidade em Transplantes do Hospital Geral de Fortaleza. Professora auxiliar I do Centro Universitário Estácio do Ceará - Fortaleza (CE), Brasil.

Enfermeira. Doutora em Enfermagem pela Universidade Federal do Ceará. Docente da Universidade de Fortaleza - Fortaleza (CE), Brasil.

*Autora correspondente: heloisasousa_nr@hotmail.com

Recebido: 03/07/2020 - Aprovado: 14/10/2020

https://doi.org/10.5327/Z1414-4425202000040005 


\section{INTRODUÇÃO}

Atualmente, Coronavirus Disease (COVID-19) é denominada uma infecção com características sintomáticas predominantemente respiratórias identificada pela primeira vez em Wuhan, na China, em dezembro de 2019. Trata-se de patologia com alto potencial de contágio. Apesar dos esforços para conter sua disseminação, a até então epidemia se propagou por vários outros países e, em 11 de março de 2020, a Organização Mundial da Saúde (OMS) declarou uma pandemia, quando já havia 118 mil casos diagnosticados em 114 países $^{1}$.

O Brasil foi o primeiro país a reportar a COVID-19 na América Latina. Desde então, até o dia 20 de junho, foram confirmados 1.067 .579 casos e 49.976 óbitos pela doença. Atualmente, é um dos países com maior incidência².

A pandemia tornou-se um grande desafio para os serviços de saúde pela quantidade de pessoas infectadas e pela demanda de recursos necessários para o seu enfrentamento, compreendendo a diversidade de materiais e profissionais envolvidos. Entre esses profissionais, encontram-se os enfermeiros, que estão na linha de frente do cuidado prestado nos diversos cenários da assistência à saúde ${ }^{3}$.

Nesse contexto, os enfermeiros configuram a maior força de trabalho para os sistemas de saúde, fundamentais para o enfretamento da COVID-19, ao considerar que esses profissionais planejam, gerenciam, avaliam e prestam cuidados em todos os níveis de complexidade ${ }^{3,4}$. Fato esse que demostra ser o enfermeiro o profissional que demanda mais tempo no cuidado direto aos pacientes.

Com relação à atuação do enfermeiro em todos os níveis de complexidade e em seus diversos campos de atuação, destaca-se que, no caso de pacientes transplantados, esses profissionais prestam cuidado especializado na proteção, promoção e reabilitação da saúde de candidatos, receptores e seus familiares ${ }^{5}$.

A enfermagem vem sendo desafiada a se adaptar e a se flexibilizar ante as mudanças e elaborar novas estratégias para minimizar os impactos gerados pela pandemia no atendimento aos pacientes transplantados, que representam um grupo de alto risco para infecção pelo coronavírus (SARSCoV2), perante sua imunossupressão crônica e suas comorbidades associadas. Assim, as instituições que atendem a essa população necessitaram reorganizar suas práticas de cuidados para esse público e tentar minimizar os riscos de adoecimento entre os pacientes transplantados ${ }^{6}$.
Nessa perspectiva, o estudo faz-se relevante, dados o crescente número de infectados pela COVID-19 e o limitado conhecimento sobre a percepção da assistência de enfermagem prestada ao paciente transplantado no atual contexto da pandemia, considerando que os profissionais da enfermagem são os que têm mais contato direto com os pacientes. Sendo assim, fornecerá subsídio à assistência de enfermagem em transplante ante a COVID-19.

\section{OBJETIVO}

Conhecer a percepção de enfermeiros que atuam em uma unidade de transplantes sobre os desafios de sua atuação ante a pandemia da COVID-19.

\section{MÉTODO}

Trata-se de um estudo de caráter descritivo e exploratório, com abordagem qualitativa, desenvolvido em uma unidade de transplantes de um hospital referência em procedimentos de alta complexidade, composta de uma unidade de terapia intensiva (UTI) pós-operatória com oito leitos e uma enfermaria pré e pós-operatória com 14 leitos. O referido hospital realiza transplantes renal, hepático, pancreático e simultâneo de rim-pâncreas e está situado em Fortaleza, no estado do Ceará.

Os indivíduos deste estudo foram convidados a participar da investigação de acordo com os seguintes critérios de inclusão: ser enfermeiro e atuar na unidade de transplantes por tempo superior a 12 meses. Excluíram-se profissionais em licença de saúde ou em gozo de férias. O fechamento amostral deu-se por saturação, ou seja, quando não havia informação nova nos depoimentos. Desse modo, participaram 14 enfermeiros.

Coletaram-se os dados no período de março a junho de 2020 , por meio de entrevista individual semiestruturada, elaborada e validada pelos próprios pesquisadores, composta de quatro perguntas norteadoras:

- "Relacionado ao fluxo de atendimento dos pacientes transplantados, como você interpreta o fluxo da UTI e da enfermaria?";

- "Qual é sua percepção sobre a distribuição de equipamentos de proteção individual (EPI) para a equipe?”;

- "Quais são os sentimentos vivenciados por você nessa pandemia?”; 
- "Pela rapidez das mudanças gerenciais no panorama da pandemia, como as orientações, por parte da gerência, estão sendo repassadas?”.

A validação do instrumento utilizado para as entrevistas seguiu as orientações de Souza et al. ${ }^{7}$, utilizando abordagens qualitativa e quantitativa como método para a validação com juízes. A qualitativa foi realizada pela avaliação por um grupo de especialistas, composto de seis profissionais da área da saúde, com titulação de especialista, mestre e doutor, e que possuíam conhecimento técnico e experiência prática em transplantes. A quantitativa, mediante o cálculo do índice de validade de conteúdo (IVC). Como resultado, obteve-se taxa de concordância entre os juízes de 0,97.

Realizaram-se as entrevistas em local privativo, sem interrupções. Os depoimentos foram gravados e transcritos de forma exaustiva, na tentativa de gerar indicadores qualitativos e quantitativos. Para garantir o anonimato, identificaram-se os enfermeiros pela letra E seguida da numeração arábica na ordem das entrevistas (E1, E2, E3...).

Analisaram-se os dados por meio do software Interface de R pour les Analyses Multidimensionnelles de Textes et de Questionnaires (IRAMUTEQ). Realizaram-se análises lexicográficas clássicas no IRAMUTEQ para compreender os dados estatísticos e quantificar as evocações e formas ${ }^{8}$. Obteve-se a classificação hierárquica descendente (CHD) para aferir os dados do dendograma em função das classes geradas, considerando as palavras $\operatorname{com} \chi^{2}>3,84(\mathrm{p}<0,05)$.

Posteriormente, realizou-se a análise fatorial por correspondência (AFC). A AFC permite, por meio de gráfico, visualizar a proximidade das palavras e das classes com base na CHD, considerando as representações no plano cartesiano, sendo outra forma de visualizar os conteúdos e as relações entre as classes. A interpretação ocorreu pela distribuição dos vocábulos com maior frequência nos quadrantes ${ }^{8}$. Por fim, gerou-se a nuvem de palavras, que unifica as palavras e dispõe graficamente em função da sua frequência.

O estudo foi submetido à apreciação do comitê de ética e pesquisa (CEP) do Hospital Geral de Fortaleza, em que foi avaliada a viabilidade de sua realização, e aprovado sob o número de parecer 4.049.919. O estudo respeitou todas as etapas das diretrizes e normas de pesquisas envolvendo seres humanos, segundo a Resolução no 466/2012 do Conselho Nacional de Saúde 9 . Foram requeridas aos enfermeiros a leitura e a assinatura do Termo de Consentimento Livre e Esclarecido (TCLE).

\section{RESULTADOS}

Compuseram a amostra 14 enfermeiros plantonistas, sendo 11 do sexo feminino e 3 do sexo masculino; todos trabalhavam há mais de um ano na referida unidade de transplantes.

O corpus geral foi constituído de 14 textos, separados em 81 segmentos de texto (ST), com aproveitamento de 69 ST (85,19\%). Emergiram 2.808 ocorrências (palavras, formas ou vocábulos), sendo 879 palavras distintas e 523 com uma única ocorrência. O conteúdo analisado foi categorizado em três classes:

- classe 1: "organização do fluxo de atendimento de casos suspeitos”, com 23 ST (33,33\%);

- classe 2: "EPI no contexto da pandemia da COVID19 ”, com 18 ST (26,09\%);

- classe 3: "o emocional ante a pandemia”, com 28 ST $(40,58 \%)$ (Figura 1).

Com o intuito de melhor ilustrar as palavras no corpus textual em suas referentes classes, organizou-se um diagrama de classes com exemplos de palavras de cada classe avaliadas por meio do teste $\chi^{2}$. Nele emergem as evocações que apresentam vocabulário semelhante entre si e vocabulário diferente das demais classes. Em seguida, serão apresentadas, operacionalizadas e exemplificadas cada uma das classes encontradas por meio da análise de CHD (Figura 2).

\section{Classe 1: "Organização do fluxo de atendimento de casos suspeitos"}

Compreende $33,33 \%$ ( $f=23 \mathrm{ST}$ ) do corpus total analisado. Constituída de palavras e radicais no intervalo entre $\chi^{2}=5,28$ (rápido) e $\chi^{2}=16,59$ (paciente). Composta de palavras como "paciente" $\left(\chi^{2}=16,59\right)$, "fluxo" $\left(\chi^{2}=16,27\right)$, "limpo" $\left(\chi^{2}=13,84\right)$, "UTI" $\left(\chi^{2}=9,62\right)$, "correto" $\left(\chi^{2}=8,49\right)$, "teste" $\left(\chi^{2}=7,39\right)$, "positivo" $\left(\chi^{2}=7,39\right)$ e "contaminar" $\left(\chi^{2}=6,27\right)$.

Essa classe se refere à percepção dos enfermeiros com relação ao fluxo de atendimento interno, manejo diagnóstico e terapêutico de pacientes com suspeita de infecção respiratória causada pela COVD-19 no contexto da unidade. Evidencia-se que não existe uma padronização das ações para o enfrentamento da pandemia. Os relatos demostraram que há desorganização de fluxos assistenciais e de processo de trabalho, aumentando, assim, os riscos à saúde ocupacional e dos pacientes: "Não tem como ter um controle efetivo para garantir uma unidade totalmente limpa. Teria que 


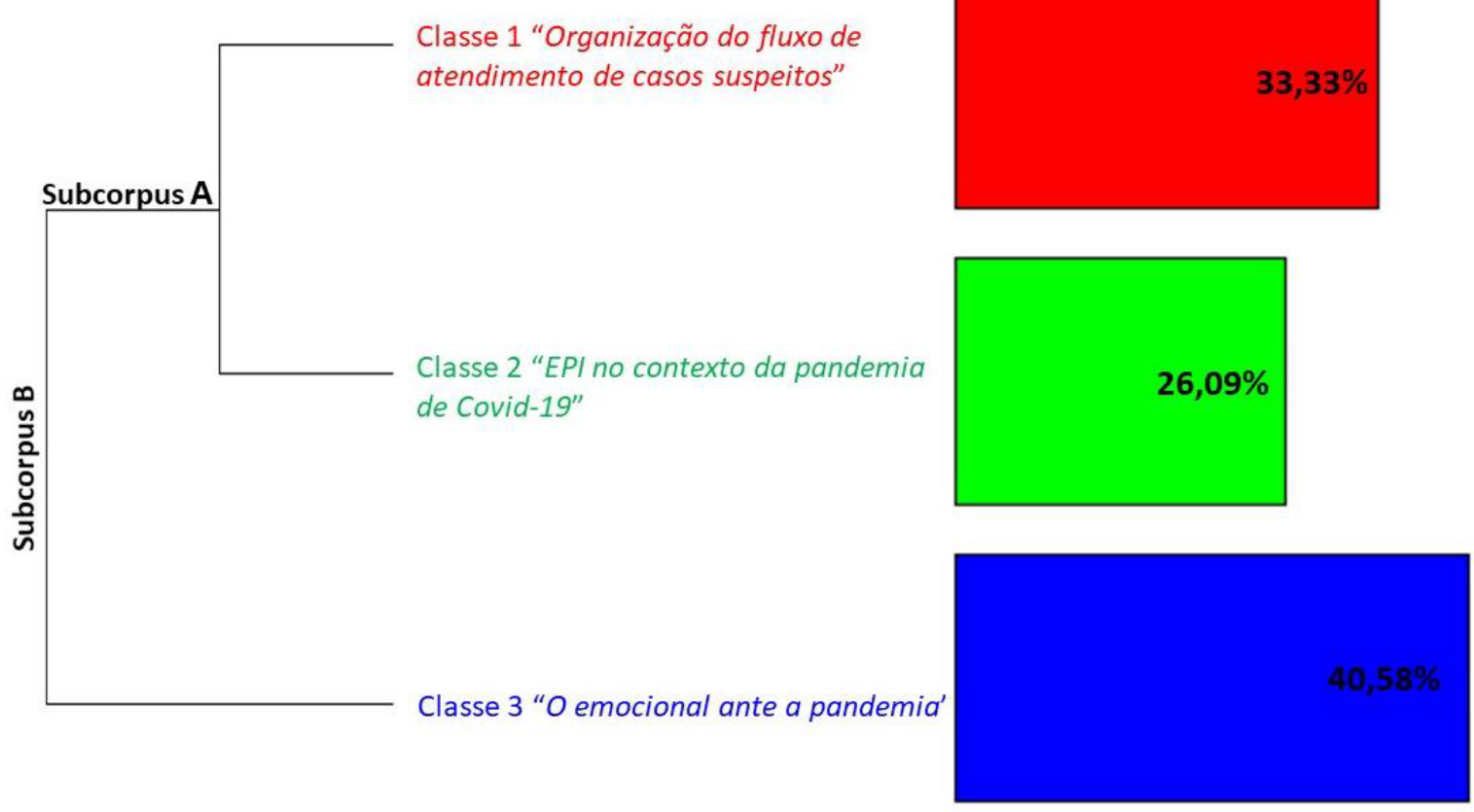

Fonte: Software IRAMUTEQ, versão: 0.7 alpha2.

Figura 1. Dendrograma da Classificação Hierárquica Descendente (CHD).

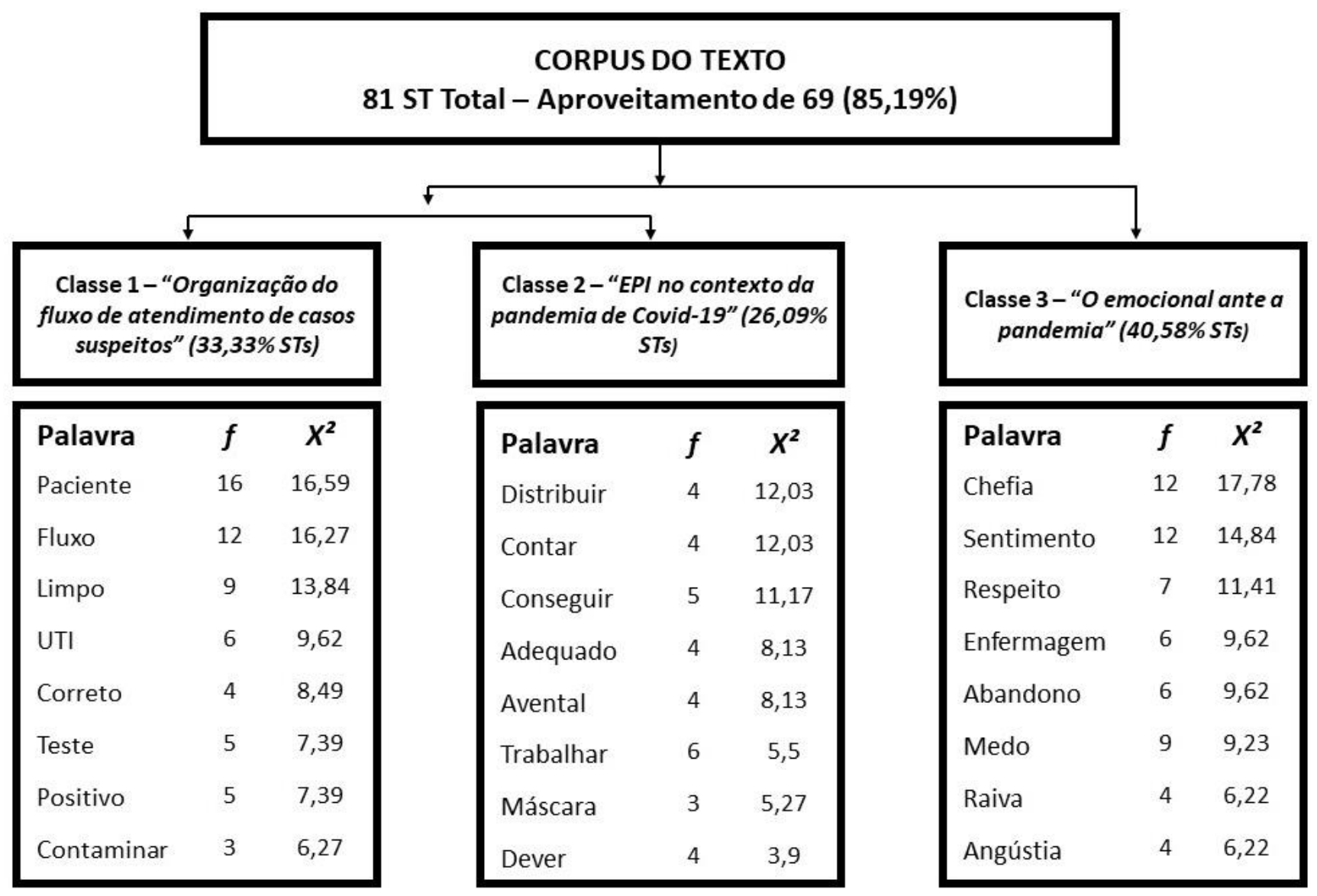

Figura 2. Organograma de classes com base nas entrevistas com os enfermeiros. 
haver dois tipos de fluxos: fluxo relacionado ao paciente [...] e fluxo relacionado ao profissional" (E2); "Eu vejo um fluxo totalmente inadequado, pois o paciente que chega na UTI nem sempre vem com o exame negativo [...], não tem um fluxo correto a seguir, coerente, de início, meio e fim" (E5); "Na verdade, a UTI clínica não é limpa, só na teoria, pois vários pacientes ao longo dos dias positivaram pra COVID, alguns foram transferidos para lá apenas com teste rápido negativo, e, quando feita a tomografia, foi dado o diagnóstico claro e conciso" (E7).

\section{Classe 2: "EPI no contexto da pandemia da COVID-19"}

Compreende $26,09 \%$ ( $f=18$ ST) do corpus total analisado. Constituída de palavras e radicais no intervalo entre $\chi^{2}=3,9$ (dever) e $\chi^{2}=12,03$ (distribuir). Composta de palavras como "distribuir" $\left(\chi^{2}=12,03\right)$, "contar" $\left(\chi^{2}=12,03\right)$, "conseguir" $\left(\chi^{2}=11,17\right)$, "adequado" $\left(\chi^{2}=8,13\right)$, "avental" $\left(\chi^{2}=8,13\right)$, "trabalhar" $\left(\chi^{2}=5,5\right)$, "máscara" $\left(\chi^{2}=5,27\right)$ e "dever" $\left(\chi^{2}=3,9\right)$.

Essa classe traz questões relacionadas à distribuição de EPI na unidade, que, no caso, não é considerada pelos órgãos competentes uma unidade de atendimento de pacientes acometidos pela COVID-19. Os depoimentos expõem a falta persistente desses equipamentos, e, quando são fornecidos, há desigualdade na distribuição, muitas vezes sem a garantia de sua efetividade, nem sempre alcançando, de forma suficiente, a segurança e a proteção ocupacional da equipe: "Os EPI deveriam ser distribuídos como em uma área COVID [...], mas só são fornecidos em parte quando há suspeita ou confirmação" (E3); "Só é distribuído EPI para setor COVID. Cada plantão enfrentamos uma batalha para conseguirmos EPI e garantirmos o mínimo de proteção para nós e os pacientes" (E4); "Os aventais são finos e passam longe de ser impermeáveis, e sapatos não são fornecidos pelo serviço. A máscara N95 é uma briga pra conseguir" (E9).

\section{Classe 3: "O emocional ante a pandemia"}

Compreende $40,58 \%$ ( $f=28$ ST) do corpus total analisado. Constituída de palavras e radicais no intervalo entre $\chi^{2}=4,2$ (pandemia) e $\chi^{2}=17,78$ (chefia). Composta de palavras como "chefia" $\left(\chi^{2}=17,78\right)$, "sentimento" $\left(\chi^{2}=14,84\right)$, "respeito" $\left(\chi^{2}=11,41\right)$, "enfermagem" $\left(\chi^{2}=9,62\right)$, "abandono" $\left(\chi^{2}=9,62\right)$, "medo" $\left(\chi^{2}=9,23\right)$, "raiva" $\left(\chi^{2}=6,22\right)$ e "angústia" $\left(\chi^{2}=6,22\right)$.

Essa classe aborda aspectos relacionados aos sentimentos vivenciados pelos profissionais na pandemia. Essa situação fez surgir diferentes reações emocionais, como medo, angústia, solidão ou raiva, assim relatadas nos depoimentos: "Sentimento de falta de respeito, abandono, frustração e medo. Preciso trabalhar para ganhar e então me submeto" (E11); "Sentimento de tristeza, abandono, falta de respeito, medo, nem sei verbalizar tamanha indignação que sinto. [...] Trabalhando em condições inadequadas" (E13).

Percebe-se que, ao relatarem suas relações de trabalho e de interação com a chefia de enfermagem, a maioria dos profissionais sente-se desvalorizada, evidenciado pelos sentimentos de abandono e de insatisfação: "Nessa pandemia, eu senti um total abandono, a falta de cuidado para conosco, o zero respeito, [...] a 'marcação' por parte da chefia em quem questiona e se posiciona sobre os acontecimentos" (E7); "Fluxo absurdamente inadequado. O setor está totalmente contaminado. Não sabemos quem propõe tamanha incoerência, mas lamentamos a aceitação da chefia [...]; sinto uma falta de respeito com o profissional” (E14).

\section{Análise fatorial por correspondência}

Com base na AFC, foi possível fazer a associação do texto entre as palavras, considerando a frequência de incidência de palavras e as classes, representando-as em um plano cartesiano (Figura 3). Realizaram-se a distribuição e a organização das palavras nos quadrantes por meio da frequência, análise esta que permite a disposição num espaço bidimensional dos elementos textuais, em função das classes geradas com base no corpus textual ${ }^{8}$.

No quadrante superior esquerdo, estão em destaque "fluxo", "paciente", "limpo" e "UTI", referentes à classe 1. No quadrante oposto superior, encontra-se a classe 3, e destacam-se as palavras "chefia", "sentimento", "abandono" e "respeito". Nesse sentido, há predominância nos discursos sobre os sentimentos e o fluxo de atendimento dos pacientes. Em oposição, nos quadrantes inferiores, encontra-se, majoritariamente, a classe 2, destacando-se as palavras "conseguir", "avental" e "máscara".

\section{Nuvem de palavras}

Em seguida, analisou-se a nuvem de palavras obtida por meio dos discursos dos participantes, na qual verifica-se que as palavras mais evocadas foram: "paciente" ( $f=33)$, "EPI" ( $f=26)$, "fluxo" ( $f=23)$, "COVID” ( $f=21)$, "UTI" $(f=14)$, "limpo" $(f=14)$, "trabalhar" $(f=13)$, "distribuição" $(f=12)$, "unidade" $(f=12)$ e "setor" $(f=10)$ (Figura 4). 


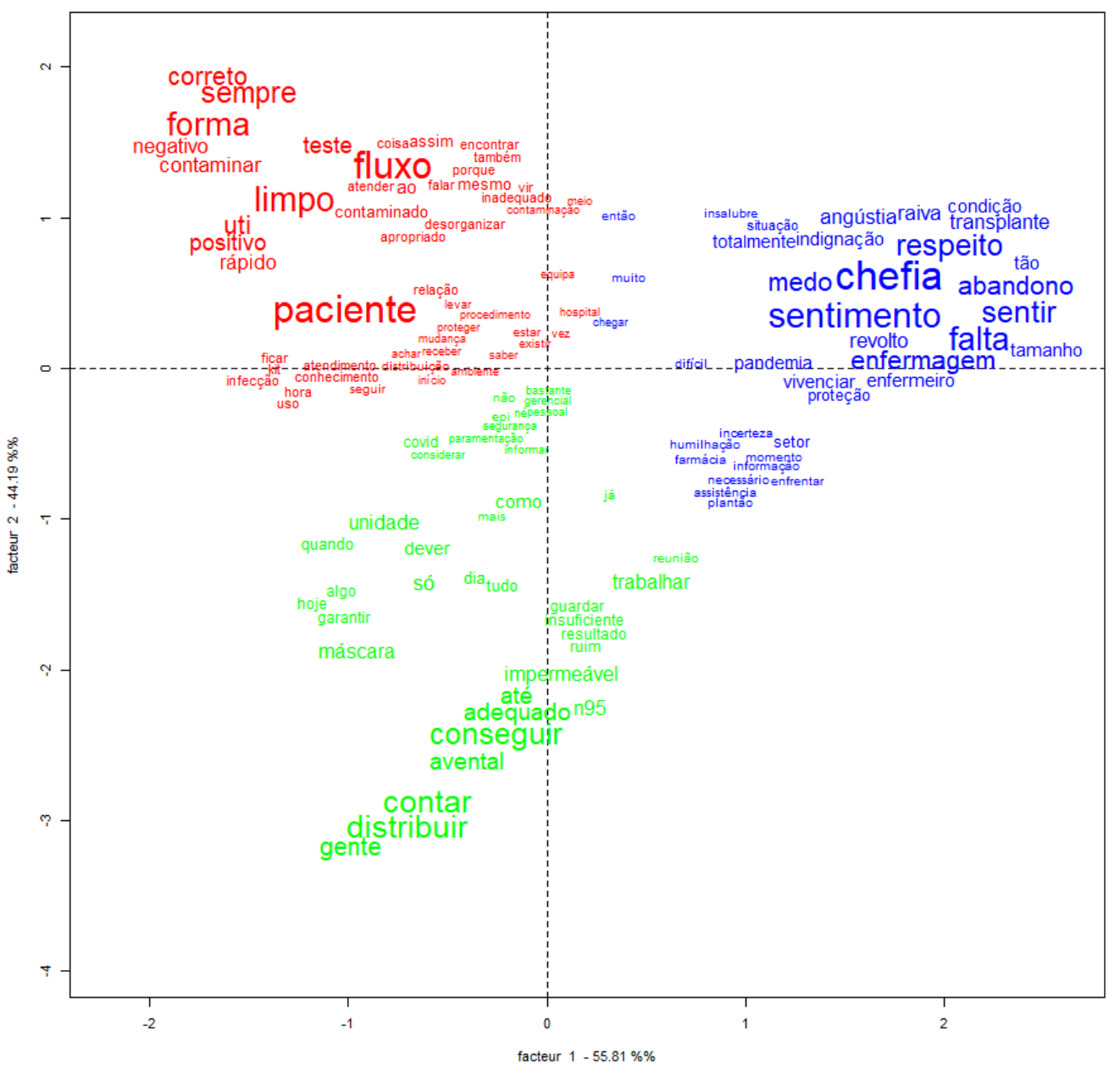

Fonte: Software IRAMUTEQ, versão: 0.7 alpha2.

Figura 3. Análise fatorial por correspondência.

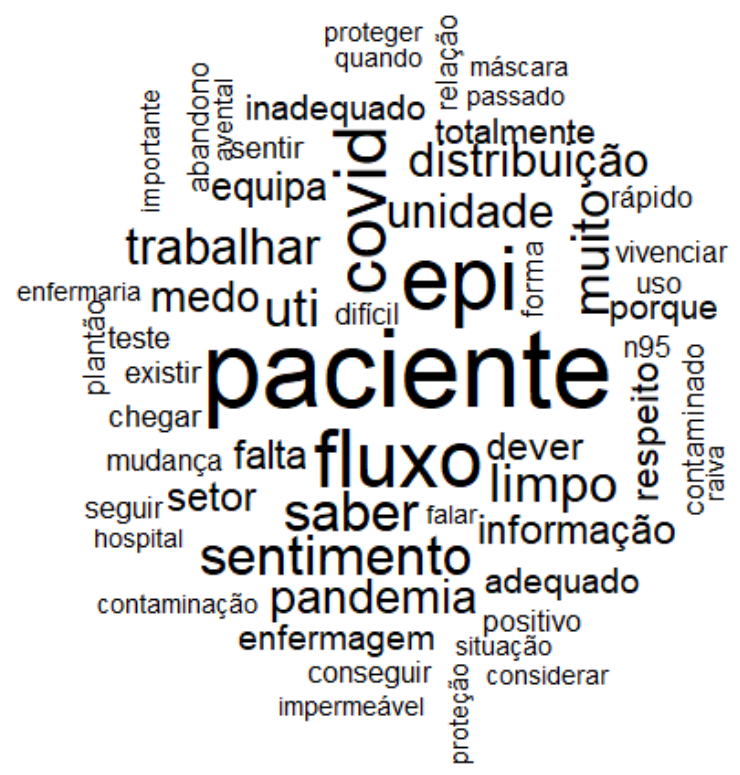

Fonte: Software IRAMUTEQ, versão: 0.7 alpha2.

Figura 4. Nuvem de palavras.

\section{DISCUSSÃO}

Os enfermeiros, entre os demais profissionais de saúde, estão diretamente envolvidos no enfrentamento da pandemia da COVID-19. Tais profissionais foram submetidos, de forma súbita, às transformações ocorridas nas rotinas dos serviços de saúde, deparando-se com unidades superlotadas, equipamentos escassos, maximizando os riscos de infecção pelo coronavírus. Nesse contexto, as condições e a organização de trabalho devem ser amplamente discutidas ${ }^{10,11}$.

Evidenciou-se, neste estudo, a insatisfação dos enfermeiros com relação ao fluxo de atendimento interno, no qual relataram desorganização nos fluxos assistenciais e no processo de trabalho. É fundamental entender a necessidade pela qual os serviços devem dispor de planejamentos operacionais e de rotina que sirvam de alerta para casos suspeitos ou confirmados de infecção pelo novo coronavírus ${ }^{12}$. 
Para assegurar condições laborais que minimizem a disseminação do vírus, medidas organizacionais devem ser elaboradas no âmbito de cada serviço de saúde ${ }^{10}$. Os gestores desses serviços possuem a atribuição de dispor de planos de ações a serem desenvolvidos, com recomendações e construções de protocolos, que podem ser modificados ao longo de tempo, devendo existir atualizações técnicas por parte da gestão e dos profissionais de saúde, com base em evidências científicas ${ }^{13}$.

Nessa perspectiva, torna-se fundamental a função dos EPI na cadeia de ações para a proteção dos profissionais de saúde na atual pandemia, evitando ou minimizando os riscos de infecção pela COVID-19 $9^{13}$. No entanto, assim como evidenciado nas falas dos participantes deste estudo, deparamo-nos com relatos de profissionais de saúde, com jornadas intensas de dedicação no cuidado beira-leito, com escassez de EPI adequados nos serviços de saúde e com a falta de garantia da efetividade desses equipamentos, quando distribuídos ${ }^{10}$. Dados que divergem da recomendação da legislação trabalhista brasileira, pela Norma Regulamentadora de Segurança e Saúde no Trabalho em Serviços de Saúde (NR32), que indica a obrigatoriedade do empregador de dispor aos seus trabalhadores EPI em quantidade suficiente, necessários para o desenvolvimento de suas atividades de forma segura, além de oferecer capacitação contínua e garantia da proteção aos profissionais sempre que houver mudança das condições de exposição ${ }^{14}$.

Ante essa escassez de EPI, nota-se, também, uma vulnerabilidade por parte desses profissionais com relação a questões emocionais, evidenciada, principalmente, pelo sentimento de medo relatado pelos enfermeiros deste estudo. Torna-se inevitável a fragilidade dos profissionais que estão na linha de frente, pois estes lidam com diversos sentimentos, entre eles: medo de contrair e transmitir o vírus, impotência, estresse, incertezas sobre a doença e o tratamento ${ }^{15}$.

O medo é compreendido como uma reação natural do ser humano ante uma ameaça real e eminente. Nesse contexto, necessita-se de um agir racional em seu enfretamento para que medidas de proteção sejam tomadas. No entanto, situações como a atual pandemia tornam o sentimento do medo constante e podem, eventualmente, gerar ansiedade em diferentes graus ${ }^{16}$.

Outro aspecto que merece atenção é a falta de valorização desses profissionais por parte da chefia de enfermagem, relatada pela maioria dos entrevistados. Cabe à gerência de enfermagem desempenhar seu papel de liderança, atendendo às diversas demandas provenientes da pandemia com o envolvimento dos demais segmentos do serviço de saúde, além de promover a contínua capacitação da equipe para a garantia da saúde desses profissionais ${ }^{17}$.
Nesse contexto, com base na AFC, no eixo Y, precisamente localizado nos quadrantes superiores, emergem assuntos acerca do fluxo de atendimento e os sentimentos vivenciados ante a pandemia. As mudanças dos fluxos de atendimento de pacientes, acompanhantes e profissionais devem ser de responsabilidade dos gestores, bem como preservar e zelar pela saúde dos profissionais da saúde ${ }^{17}$. Ainda assim, estudos relatam que profissionais expostos diretamente aos riscos de contaminação apresentaram ansiedade, irritabilidade, exaustão, entre outros sentimentos negativos $^{18}$. Já o eixo X engloba assuntos que se referem ao acesso aos EPI. Esse acesso se tornou uma constante preocupação, por sua escassez em diversas instituições de saúde do Brasil e do mundo ${ }^{19}$.

Ante tantos aspectos, o fortalecimento da interdisciplinaridade e uma comunicação eficaz entre as equipes tornam-se mais importantes do que nunca, pois, além das atividades assistenciais e administrativas, os profissionais precisam acolher, confortar e apoiar o paciente que já lida com diversas batalhas e, atualmente, enfrenta essa temorosa pandemia ${ }^{17}$. Observou-se na nuvem de palavras uma grande frequência na evocação da palavra paciente, ressaltando a importância de um atendimento de qualidade a ele.

Como limitações deste estudo, podemos citar a forma resumida pela qual os depoimentos foram concedidos pelos enfermeiros, pois as entrevistas ocorreram, em sua maioria, durante a rotina de trabalho, e os participantes poderiam estar atentos à duração da entrevista. Além disso, há a escassez de pesquisas relacionadas ao tema, impossibilitando o comparativo dos resultados com estudos similares.

\section{CONCLUSÃO}

O desenvolvimento do presente estudo possibilitou conhecer a percepção dos enfermeiros que atuam em uma unidade de transplantes dada a alteração de sua atuação na pandemia da COVID-19. Constatou-se que os enfermeiros sofrem ante as fragilidades relacionadas ao momento vivenciado, assim como os demais profissionais da saúde, sendo cotidianamente desafiados a se adaptarem às mudanças em suas rotinas de trabalho no cuidado ao paciente transplantado.

Faz-se necessário rever tal situação e desenvolver estratégias para garantir a assistência segura pela enfermagem, de modo que haja reorganização contínua e dinâmica de fluxos de admissão ao paciente na unidade, com participação da equipe e melhoria na condição de trabalho. Assim, será possível a redução dos efeitos deletérios à saúde desses profissionais e de seus pacientes ante a atual pandemia. 


\section{REFERÊNCIAS}

1. Frater JL, Zini G, d'Onofrio G, Rogers HJ. COVID-19 and the clinical hematology laboratory. Int J Lab Hematol. 2020;42(S1):11-8. https://doi. org/10.1111/ijlh.13229

2. Brasil. Ministério da Saúde (MS). Boletim epidemiológico especial. Doença pelo Coronavírus [Internet]. 2020 [acesso em 5 ago. 2020]. Disponível em: http://antigo.saude.gov.br/images/pdf/2020/July/01/Boletimepidemiologico-COVID-20-3.pdf

3. Miranda FMD, Santana LL, Pizzolato AC, Sarquis LMM. Condições de trabalho e o impacto na saúde dos profissionais de enfermagem frente à Covid-19. Cogitare Enferm. 2020;25:e72702. http://doi.org/10.5380/ ce.v25i0.72702

4. Jackson D, Bradbury-Jones C, Baptiste D, Gelling L, Morin K, Neville S, et al. Life in the pandemic: some reflections on nursing in the context of COVID-19. J Clin Nurs. 2020;29(13-14):2041-3. https://doi.org/10.1111/ jocn. 15257

5. Marques RVS, Freitas VL. Importance of nursing care in renal transplanted patient care. J Nursing UFPE online [Internet]. 2018 [acesso em 5 ago. 2020];12(12):3436-44. Disponível em: https://periodicos.ufpe. $\mathrm{br} /$ revistas/revistaenfermagem/article/view/237692 https://doi. org/10.5205/1981-8963-v12i12a237692p3436-3444-2018

6. Knihs NS, Sens S, Silva AM, Wachholz LF, Paim SMS, Magalhães ALP. Transição do cuidado de pacientes submetidos ao transplante hepático durante a pandemia da Covid-19. Texto Contexto Enferm [Internet]. 2020 [acesso em 15 ago. 2020];29:e20200191. Disponível em: https://www. scielo.br/pdf/tce/v29/pt_1980-265X-tce-29-e20200191.pdf https://doi. org/10.1590/1980-265X-TCE-2020-0191

7. Souza AC, Alexandre NMC, Guirardello EB. Propriedades psicométricas na avaliação de instrumentos: avaliação da confiabilidade e da validade. Epidemiol Serv Saúde. 2017;26(3):649-59. https://doi.org/10.5123/ s1679-49742017000300022

8. Souza MAR, Wall ML, Thuler ACMC, Lowen IMV, Peres AM. The use of IRAMUTEQ software for data analysis in qualitative research. RevEsc Enferm USP. 2018;52:e03353. https://doi.org/10.1590/s1980-220×2017015003353

9. Brasil. Ministério da Saúde (MS). Conselho Nacional de Saúde. Resolução n 466, de 12 de dezembro de 2012. Dispõe sobre diretrizes e normas regulamentadoras de pesquisas envolvendo seres humanos [Internet]. Brasília: Conselho Nacional de Saúde; 2013 [acesso em 19 set. 2019]. Disponível em: https://conselho. saude.gov.br/resolucoes/2012/Res0466.pdf
10. Oliveira AC. Desafios da enfermagem frente ao enfrentamento da pandemia da Covid19. Rev Min Enferm. 2020;24:e-1302. http://doi. org/10.5935/1415-2762.20200032

11. Jackson Fiho JM, Assunção AA, Algranti E, Garcia EG, Saito CA, Maeno M. A saúde do trabalhador e o enfrentamento da COVID-19. Rev Bras Saúde Ocup. 2020;45:10-2. https://doi. org/10.1590/2317-6369ed0000120

12. Gallasch CH, Cunha ML, Pereira LAS, Silva-Junior JS. Prevenção relacionada à exposição ocupacional do profissional de saúde no cenário de COVID-19. Rev Enferm UERJ. 2020;28:49596. http://doi. org/10.12957/reuerj.2020.49596

13. Almeida IM. Proteção da saúde dos trabalhadores da saúde em tempos de COVID-19 e respostas à pandemia. Rev Bras Saúde Ocup. 2020;45:1-10. http://doi.org/10.1590/scielopreprints. 140

14. Brasil. Ministério do Trabalho e Emprego. Portaria n 485, de 11 de Novembro de 2005. Diário Oficial da União [Internet]. 2005 [acesso em 5 ago. 2020];1:29. Disponível em: http://sbbq.iq.usp.br/arquivos/ seguranca/portaria485.pdf

15. Saidel MGB, Lima MHDM, Campos CJG, Loyola CMD, Espiridião $\mathrm{E}$, Rodrigues J. Intervenções em saúde mental para profissionais de saúde frente à pandemia de coronavírus. Rev Enferm UERJ. 2020;28:e49923. https://doi.org/10.12957/reuerj.2020.49923

16. Silva HGN, Santos LES, Oliveira AKS. Efeitos da pandemia do novo coronavírus na saúde mental de indivíduos e coletividades. J Nurs Heal. 2020 [acesso em 15 ago. 2020]; 10(N. Esp.):e20104007. Disponível em: https://periodicos.ufpel.edu.br/ojs2/index.php/enfermagem/ article/view/18677/11414

17. Ramos RS. A enfermagem oncológica no enfrentamento da pandemia de Covid-19: reflexões e recomendações para a prática de cuidado em oncologia. Rev Bras Cancerol. 2020;66:e1007. https://doi. org/10.32635/2176-9745.RBC.2020v66nTemaAtual.1007

18. Medeiros EAS. A luta dos profissionais de saúde no enfrentamento da COVID-19. Acta Paul Enferm. 2020;33:e-EDT2020003. http://doi. org/10.37689/acta-ape/2020edt0003

19. Cruz RM, Borges-Andrade JE, Moscon DCB, Micheletto MRD, Esteves GGL, Delben PB, et al. COVID-19: emergência e impactos na saúde e no trabalho. Rev Psicol Organ e Trab. 2020;20(2):I-III. https://doi. org/10.17652/rpot/2020.2.editorial 